\title{
Ingestão de bebidas alcoólicas em lactantes atendidas em Hospital Universitário
}

\author{
Alcohol intake in lactating women assisted in a University Hospital
}

\begin{abstract}
Ana Luisa V. Nascimento ${ }^{1}$, Amanda Fernandes O. de Souza ${ }^{1}$, Ana Carolina R. de Amorim¹, Mayara Brasil de S. Leitão', Regiane Maio², Maria Goretti P. A. Burgos ${ }^{3}$
\end{abstract}

\section{RESUMO}

Objetivos: Verificar a frequência do consumo de bebidas alcoólicas e o grau de risco do hábito de etilismo em lactantes atendidas no Serviço de Puericultura do Hospital das Clínicas da Universidade Federal de Pernambuco.

Métodos: Estudo transversal com 157 mães lactantes matriculadas no Programa de Puericultura do hospital. Utilizou-se questionário com informações sobre variáveis demográficas, socioeconômicas, tipo e tempo de aleitamento materno, hábito de tabagismo e consumo de alimentos considerados petiscos. A avaliação do consumo de álcool nos últimos 12 meses foi realizada pelo questionário "teste de identificação de distúrbios causados pelo uso de álcool” (AUDIT C - Alcobol Use Disorders Identification Test). Para análise estatística, aplicaram-se os testes do qui-quadrado de Pearson e exato de Fisher.

Resultados: Verificou-se consumo de bebidas alcoólicas em $12 \%$ das lactantes; dentre estas, $100 \%$ apresentaram baixo risco para transtornos causados pelo uso do álcool. A frequência de lactantes que consumiam petiscos durante a ingestão alcoólica foi $100 \%$, sendo os queijos os mais consumidos - 18 (95\%).

Conclusões: A prevalência de consumo de álcool foi baixa nas lactantes estudadas. As usuárias exibiram um consumo considerado de baixo risco e uma frequência elevada de consumo de petiscos durante a ingestão alcoólica.

Palavras-chave: bebidas alcoólicas; aleitamento materno; nutrição materna; nutrição do lactente.

Instituição: Hospital das Clínicas da Universidade Federal de Pernambuco (UFPE), Recife, PE, Brasil

'Graduanda em Nutrição do Departamento de Nutrição da UFPE, Recife, PE, Brasil

2Doutora em Nutrição pela Universidade Estadual Paulista "Júlio de Mesquita Filho" (Unesp); Professora Adjunta do Departamento de Nutrição da UFPE, Recife, PE, Brasil

3Doutora em Nutrição pela UFPE; Professora Adjunta do Departamento de Nutrição da UFPE, Recife, PE, Brasil

\section{ABSTRACT}

Objective: To determine the prevalence of alcohol intake and the degree of alcohol-related risk among nursing mothers attended at the Child Care Service of Hospital das Clínicas of Universidade Federal de Pernambuco, Brazil.

Methods: A cross-sectional study was carried out with 157 nursing mothers enrolled in the Child Care Program of the university hospital. A questionnaire was administered addressing demographic and socioeconomic variables, type and duration of breastfeeding, smoking habits and consumption of foods considered as appetizers. The Alcohol Use Disorders Identification Test (AUDIT C) was applied for assessing alcohol consumption in the previous 12 months. Pearson's chi-square test and Fisher's exact test were used for statistical analysis.

Results: Twelve percent of the nursing mothers reported consuming alcoholic beverages, $100 \%$ of whom were classified as being at low risk for alcohol use disorders. The frequency of nursing mothers who consumed appetizers during alcohol consumption was $100 \%$, the most common of which was cheese - 18 (95\%).

Conclusions: The prevalence of alcohol intake was low in the nursing mothers analyzed. The users exhibited a low risk for alcohol disorders and a high frequency of the consumption of appetizers during alcohol consumption.

Key-words: alcoholic beverages; breast feeding; maternal nutrition; infant nutrition.

Endereço para correspondência:

Ana Luisa Valadares Nascimento

Rua General Joaquim Inácio, 170, apto 05 - Ilha do Leite

CEP 50070-270 - Recife/PE

E-mail: nutrianaluisa@gmail.com

Fonte financiadora: Fundação de Amparo à Ciência e Tecnologia do Estado de Pernambuco, protocolo: BIC - 0915-4.05/11

Conflito de interesse: nada a declarar

Recebido em: 23/7/2012

Aprovado em: 15/1/2013 


\section{Introdução}

A lactação é uma das funções fisiológicas mais antigas entre os mamíferos e, cada vez mais, o leite materno (LM) é considerado de vital importância para o recém-nascido $(\mathrm{RN})^{(1)}$. No Brasil, as últimas estatísticas acerca do aleitamento materno são do Programa Nacional de Saúde e Nutrição $(\mathrm{PNSN})^{(2)}$ e indicam que $99,5 \%$ dos RN mamam logo após o nascimento, e a duração da alimentação exclusiva com LM subiu de um para 2,2 meses. O aleitamento materno é a melhor prática a ser adotada em benefício do lactente nos primeiros seis meses de vida, seja sob o ponto de vista nutricional ou quanto à proteção contra doenças, além de aspectos emocionais e condições sociais ou econômicas.

Ao oferecer seu leite, a mulher transfere diversos nutrientes à criança, visando o crescimento e o bem-estar do lactente. Porém, circunstancialmente, o leite materno também pode servir como veículo de substâncias nocivas, como em algumas doenças infecciosas, nas quais é necessário o uso de fármacos para o tratamento, bem como no consumo de drogas como o álcool, que, embora não esteja incluído na relação das substâncias que contraindicam o aleitamento materno, pode causar prejuízos tanto para a criança quanto para a nutriz ${ }^{(3,4)}$.

Vários autores já evidenciaram, em estudos com animais, que o álcool passa através do leite, alterando a produção, o volume, o aroma, a composição e a excreção láctea, além de provocar efeitos deletérios no $\mathrm{RN}^{(5-7)}$. O consumo de álcool pela lactante altera os ciclos de vígília-sono do RN. Estudos mostram que a criança consome aproximadamente 20\% menos leite durante as horas imediatamente após a ingestão de uma dose aguda de bebidas alcoólicas pela mãe $e^{(8,9)}$. Essa alteração no consumo de LM deve-se, em parte, à diminuição na produção de leite, bem como à modificação de seu odor após a ingestão alcoólica. Assim, o lactente, apesar de sugar de forma mais vigorosa, ingere menos leite por tempo de sucção, fato não percebido pelas mães ${ }^{(5,10)}$. Além disso, o álcool causa efeitos sobre o comportamento do bebê, interfere no sistema imunológico e no sistema nervoso central e, em longo prazo, pode comprometer o crescimento e elevar a mortalidade neonatal ${ }^{(11)}$.

A quantidade de etanol transmitida para o leite humano é uma fração diminuta do que foi ingerido pela mãe (menos de $2 \%$ da dose materna). Portanto, a exposição ocasional é, muitas vezes, considerada insignificante. Muitos orgãos, como o Comitê de Drogas da Academia Americana de Pediatria dos Estados Unidos, classificam o etanol como uma "droga normalmente permitida com a amamentação"(12).
Em países como a Austrália, bebidas alcoólicas são aceitas como parte da cultura e, por isso, são amplamente consumidas. As mulheres australianas habitualmente consomem álcool, constituindo $6 \%$ de calorias da dieta básica diária; $35 \%$ consomem semanalmente, estando a maioria delas em período de amamentação ${ }^{(13)}$.

O consumo excessivo de bebidas alcoólicas é considerado um problema mundial. Dados mais recentes da Organização Mundial da Saúde (OMS) mostram que os brasileiros consomem 18,5L de álcool puro por ano. No continente americano, o valor é menor apenas que o do Equador (29,9L), México $(27,2 \mathrm{~L})$ e Nicarágua $(20,5 \mathrm{~L})$. O Relatório Global sobre Saúde e Consumo de Álcool da OMS, de 2011, com dados referentes até o ano de 2005, mostrou que, entre as mulheres, o consumo é de 10,6L por ano. No Brasil, $54 \%$ das pessoas que bebem escolhem cerveja; $40 \%$ escolhem destilados, $5 \%$, vinho, e outros tipos de bebida somam $1 \%^{(14)}$.

Embora nos últimos anos tenha ocorrido incremento nas campanhas educativas, alertando contra os malefícios do consumo de álcool, ainda se observa que uma parcela considerável da população feminina o mantém durante a amamentação, salientando ser um hábito cultural a recomendação de algumas bebidas alcoólicas como fontes de vitaminas do complexo B e capazes de provocar o relaxamento de mãe e filho ${ }^{(4,5)}$.

Devido aos poucos achados na literatura e à ocorrência do elevado consumo de bebidas alcoólicas entre mulheres com idade fértil no mundo ocidental —e sabendo-se que o etanol é uma droga bastante ingerida na nossa região - objetivou-se, neste estudo, observar a prevalência desse hábito e o grau de risco do etilismo entre as mães de lactentes, atendidas no Ambulatório de Puericultura do Hospital das Clínicas da Universidade Federal de Pernambuco (HC-UFPE). Além disso, pretendeu-se verificar a associação desse consumo com as variáveis socioeconômicas e demográficas estudadas, bem como com hábitos durante a ingestão de álcool.

\section{Método}

Estudo transversal, no qual foram incluídas todas as lactantes que frequentavam o ambulatório no período de junho de 2011 a fevereiro de 2012. Estudaram-se 157 lactantes recrutadas de modo consecutivo nos dias do atendimento médico de rotina ou eventual. Para composição da casuística, consideraram-se os seguintes critérios de inclusão: lactantes de qualquer idade e raça e em qualquer período de amamentação. Excluíram-se aquelas com incapacidade mental para 
responder às questões do estudo. A pesquisa foi conduzida após aprovação do Comitê de Ética em Pesquisa da UFPE, em obediência à Resolução do Conselho Nacional de Saúde n¹96/96 sobre "Pesquisa envolvendo Seres Humanos". A participação no estudo foi voluntária, após obtenção do consentimento das lactantes mediante o termo de consentimento livre e esclarecido.

Para caracterizar o perfil socioeconômico e demográfico, elaborou-se um formulário que continha questões fechadas sobre faixa etária, etnia, estado civil, grau de escolaridade, paridade, ocupação, renda mensal e procedência (cidade do Recife, Região Metropolitana, interior ou outros estados), além do tipo e tempo de aleitamento materno e intervalo entre as mamadas. Cada participante que respondeu positivamente sobre a ingestão de álcool, mesmo que raramente, foi inserida no grupo das que faziam uso de bebidas alcoólicas. Para avaliar o risco da ingestão, aplicou-se o teste de identificação de distúrbios causados pelo uso de álcool (AUDIT C), validado em nível internacional ${ }^{(15)}$, que classifica o uso de álcool em: baixo risco ou abstêmios $=0-7$ pontos; consumo de risco $=8-15$ pontos; uso nocivo ou consumo de alto risco $=16-19$ pontos; provável dependência $=30$ ou mais pontos ${ }^{(16)}$. Durante a entrevista, foram feitas perguntas sobre o hábito de usar tabaco e de consumir petiscos alimentares. Para verificar o consumo de alimentos (petiscos), as lactantes foram questionadas se consumiam algum alimento durante o consumo alcoólico, sendo as possibilidades de respostas sim ou não. Na presença de resposta positiva, assinalavam-se os petiscos consumidos de modo isolado ou associados, conforme a lista: queijos, frios (salame, presunto etc.), carnes secas, salsichas, linguiças, salgados fritos, ovo de codorna, frutos do mar, amendoins e azeitonas.

Apresentaram-se as variáveis como frequências relativas, utilizando-se os testes exato de Fisher e qui-quadrado de Pearson para verificar a relação entre o consumo alcoólico e as demais variáveis estudadas, considerando-se o nível de significância de $5 \%$.

\section{Resultados}

A amostra foi de 157 lactantes, com média de idade de

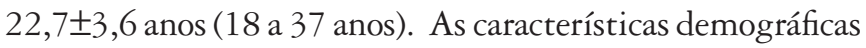
e socioeconômicas são apresentadas na Tabela 1 .

Quanto ao aleitamento materno, $130(82,8 \%)$ mães estavam amamentando por um período menor que três meses, $22(14 \%)$ de três a seis meses e cinco $(3,2 \%)$ por tempo maior que seis meses. Verificou-se aleitamento exclusivo em
$73,2 \%$ das lactantes - com intervalos entre as mamadas de $30-40 \min (51 \%), 1-2$ horas $(33,1 \%)$ ou maior que 2 horas $(15,9 \%)$ (Tabela 2$)$.

Tabela 1 - Características demográficas e socioeconômicas de lactantes atendidas no Ambulatório de Puericultura do Hospital das Clínicas da Universidade Federal de Pernambuco, Recife/ Brasil, 2011-2012

\begin{tabular}{lrc}
\hline Variável & $\mathbf{n}$ & $\%$ \\
\hline Faixa etária (anos) & 32 & 20,4 \\
$\quad$ Até 19 & 125 & 79,6 \\
$\quad \geq 20$ & & \\
Etnia & 40 & 25,5 \\
$\quad$ Branca & 117 & 74,5 \\
$\quad$ Não branca & & \\
Estado civil & 83 & 52,9 \\
$\quad$ Solteira/separada/divorciada/viúva & 74 & 47,1 \\
$\quad$ Casada/união estável & & \\
$\quad$ Escolaridade & 34 & 21,7 \\
$\quad$ Ensino fundamental & 106 & 67,5 \\
$\quad$ Ensino médio & 17 & 10,8 \\
$\quad$ Ensino superior & & \\
Ocupação & 33 & 21,0 \\
$\quad$ Sim & 124 & 79,0 \\
$\quad$ Não & & \\
Renda familiar & 61 & 38,9 \\
$\quad$ Até 1 salário & 69 & 43,9 \\
2 salários & \\
$\quad$ Mais de 2 salários & & 17,2 \\
Procedência & & \\
$\quad$ Recife & & 84,7 \\
\hline
\end{tabular}

Tabela 2 - Distribuição das lactantes segundo o tempo de aleitamento materno, tipo de aleitamento e intervalo entre as mamadas, atendidas no Ambulatório de Puericultura do Hospital das Clínicas da Universidade Federal de Pernambuco, Recife/ Brasil, 2011-2012

\begin{tabular}{lrr}
\hline Variável & $\mathbf{n}$ & $\%$ \\
\hline Tempo de aleitamento materno (meses) & & \\
$\quad<3$ & 130 & 82,8 \\
$\quad 3$ & 27 & 17,2 \\
Tipo de aleitamento materno & & \\
$\quad$ Materno exclusivo & 115 & 73,2 \\
$\quad$ Materno complementar & 42 & 26,8 \\
Intervalo entre as mamadas & & \\
30 a 40 minutos & 80 & 51,0 \\
1 a 2 horas & 52 & 33,1 \\
$>2$ horas & 25 & 15,9 \\
Total & 157 & 100,0 \\
\hline
\end{tabular}


Tabela 3 - Associação entre o hábito de etilismo e as variáveis relacionadas ao aleitamento materno em lactantes atendidas no Ambulatório de Puericultura do Hospital das Clínicas da Universidade Federal de Pernambuco, Recife/Brasil, 2011-2012

\begin{tabular}{|c|c|c|c|c|c|c|c|}
\hline \multirow{3}{*}{ Variável } & \multicolumn{4}{|c|}{ Hábito do etilismo } & \multirow{2}{*}{\multicolumn{2}{|c|}{ Total }} & \multirow{3}{*}{ Valor $p$} \\
\hline & \multicolumn{2}{|c|}{ Sim } & \multicolumn{2}{|c|}{ Não } & & & \\
\hline & $\mathbf{n}$ & $\%$ & $\mathbf{n}$ & $\%$ & $\mathbf{n}$ & $\%$ & \\
\hline \multicolumn{8}{|c|}{ Tempo de aleitamento materno (meses) } \\
\hline$<3$ & 14 & 73,7 & 116 & 84,1 & 130 & 82,8 & 0,327 \\
\hline$\geq 3$ & 5 & 26,3 & 22 & 15,9 & 27 & 17,2 & \\
\hline \multicolumn{8}{|l|}{ Tipo de aleitamento materno } \\
\hline Materno exclusivo & 11 & 57,9 & 104 & 75,4 & 115 & 73,2 & 0,107 \\
\hline Materno complementar & 8 & 42,1 & 34 & 24,6 & 42 & 26,8 & \\
\hline \multicolumn{8}{|c|}{ Intervalo entre as mamadas (horas) } \\
\hline Até 2 & 16 & 84,2 & 116 & 84,1 & 132 & 84,1 & 1,000 \\
\hline$\geq 2$ & 3 & 15,8 & 22 & 15,9 & 25 & 15,9 & \\
\hline Total & 19 & 100,0 & 138 & 100,0 & 157 & 100,0 & \\
\hline
\end{tabular}

Com relação ao consumo de álcool, $19(12,1 \%)$ lactantes responderam afirmativamente e $100 \%$ do grupo consumiam alimentos durante a ingestão alcoólica, sendo a cerveja, a vodka e o vinho as bebidas preferidas (100, 36,8 e 21,1\%, respectivamente). Pelo teste AUDIT C, todo o grupo foi classificado como baixo risco/abstêmios para transtornos causados pelo uso do álcool.

Para o consumo de alimentos durante a ingestão de bebidas, verificou-se que $18(94,7 \%)$ lactantes consumiam queijos; $17(89,5 \%)$, caldo de leguminosas; $17(89,5 \%)$, amendoim; 16 (84,2\%), ovos; 15 (78,9\%), azeitonas; 14 $(73,7 \%)$, consumiam frios; $13(68,4 \%)$, frutos do mar; 12 $(63,2 \%)$, carne seca; $12(63,2 \%)$, embutidos e $9(47,4 \%)$, salgados fritos.

As associações entre o consumo de bebidas alcoólicas e as variáveis relacionadas ao aleitamento, demográficas e socioeconômicas encontram-se nas Tabelas 3 e 4, respectivamente. Nenhuma associação estatisticamente significante foi observada.

\section{Discussão}

Embora o álcool seja reconhecido como deletério à saúde humana, seu consumo ainda se faz presente no dia a dia da população brasileira, verificando-se, em estudo recente, consumo excessivo de álcool em $18 \%$ das mulheres ${ }^{(17)}$. É comum o uso de álcool durante a gravidez ou lactação, apesar de as diretrizes governamentais nacionais e internacionais recomendarem que o mesmo não deva ser consumido nos períodos pré e pós-natal ${ }^{(17,18)}$.
O hábito de consumir bebidas alcoólicas ocorre em mulheres que estão amamentando, com frequências que variam de 36 a $80 \%{ }^{(4)}$. No presente estudo, encontrou-se frequência menor $(12,1 \%)$ de consumo de bebidas alcoólicas - valor semelhante ao referido por Del Ciampo et $a l^{(4)}$, em estudo com 504 mulheres paulistas, cuja prevalência foi de $11,1 \%$. Assim, os estudos publicados mostram diferenças consideráveis na frequência de consumo alcoólico em lactantes. $\mathrm{Na}$ amostra estudada, todas as mulheres que consumiam álcool foram classificadas como sendo de baixo risco para transtornos causados pelo uso da substância, o que foi similar ao relatado por Del Ciampo et al, em $2009^{(4)}$. Reconhecidamente, a ingestão de álcool não é recomendada durante a amamentação. O Ministério da Saúde alerta para seu uso criterioso, uma vez que a ingestão de doses iguais ou maiores que $0,3 \mathrm{~g} / \mathrm{kg}$ de peso podem reduzir a produção láctea, modificar o odor e o sabor do leite materno, levando à recusa do mesmo pelo lactente ${ }^{(19)}$.

No México, mulheres em fase de aleitamento são encorajadas a beber cerca de $2 \mathrm{~L}$ diários de pulque, bebida fermentada que possui uma pequena porcentagem alcoólica ${ }^{(20)}$. Na Alemanha, a cerveja é considerada o "elixir mágico", enquanto a comunidade indochinesa na Califórnia prefere ervas medicinais embebidas em vinho ${ }^{(21)}$. No Brasil, a cultura popular também reconhece a ingestão de cervejas mais escuras e "fortificadas". Por não serem filtradas, é fato que contêm mais proteínas e lúpulo ${ }^{(22,23)}$. Em vários povos, persiste a crença de que o álcool seria galactogênico, ou seja, o consumo de pequenas quantidades imediatamente antes do aleitamento facilitaria a produção de leite nas glândulas mamárias. Logo 
Tabela 4 - Associação entre o hábito de etilismo e as variáveis demográficas e socioeconômicas em lactantes atendidas no Ambulatório de Puericultura do Hospital das Clínicas da Universidade Federal de Pernambuco, Recife/Brasil, 2011-2012

\begin{tabular}{|c|c|c|c|c|c|c|c|}
\hline \multirow{3}{*}{ Variável } & \multicolumn{4}{|c|}{ Hábito do etilismo } & \multirow{2}{*}{\multicolumn{2}{|c|}{ Total }} & \multirow{3}{*}{ Valor $p$} \\
\hline & \multicolumn{2}{|c|}{ Sim } & \multicolumn{2}{|c|}{ Não } & & & \\
\hline & $\mathrm{n}$ & $\%$ & $\mathrm{n}$ & $\%$ & $\mathrm{n}$ & $\%$ & \\
\hline \multicolumn{8}{|l|}{ Faixa etária (anos) } \\
\hline$<20$ & 4 & 12,5 & 28 & 87,5 & 32 & 100,0 & \multirow{3}{*}{0,303} \\
\hline 20 a 24 & 13 & 15,1 & 73 & 84,9 & 86 & 100,0 & \\
\hline$\geq 25$ & 2 & 5,1 & 37 & 94,9 & 39 & 100,0 & \\
\hline \multicolumn{8}{|l|}{ Etnia } \\
\hline Branca & 3 & 7,5 & 37 & 92,5 & 40 & 100,0 & \multirow[t]{2}{*}{0,405} \\
\hline Não branca & 16 & 13,7 & 101 & 86,3 & 117 & 100,0 & \\
\hline \multicolumn{8}{|l|}{ Estado civil } \\
\hline Solteira/separada/divorciada/viúva & 8 & 9,6 & 75 & 90,4 & 83 & 100,0 & \multirow[t]{2}{*}{0,316} \\
\hline Casada/união estável & 11 & 14,9 & 63 & 85,1 & 74 & 100,0 & \\
\hline \multicolumn{8}{|l|}{ Escolaridade } \\
\hline Ensino fundamental & 1 & 2,9 & 33 & 97,1 & 34 & 100,0 & \multirow{3}{*}{0,145} \\
\hline Ensino médio & 16 & 15,1 & 90 & 84,9 & 106 & 100,0 & \\
\hline Ensino superior & 2 & 11,8 & 15 & 88,2 & 17 & 100,0 & \\
\hline \multicolumn{8}{|l|}{ Ocupação } \\
\hline $\operatorname{Sim}$ & 4 & 12,1 & 29 & 87,9 & 33 & 100,0 & \multirow[t]{2}{*}{1,000} \\
\hline Não & 15 & 12,1 & 109 & 87,9 & 124 & 100,0 & \\
\hline \multicolumn{8}{|l|}{ Renda (salários mínimos) } \\
\hline Até um & 8 & 13,1 & 53 & 86,9 & 61 & 100,0 & \multirow{3}{*}{0,713} \\
\hline Dois & 9 & 13,0 & 60 & 87,0 & 69 & 100,0 & \\
\hline Mais de dois & 2 & 7,4 & 25 & 92,6 & 27 & 100,0 & \\
\hline \multicolumn{8}{|l|}{ Procedência } \\
\hline Recife & 16 & 12,0 & 117 & 88,0 & 133 & 100,0 & \multirow[t]{2}{*}{0,874} \\
\hline Região Metropolitana do Recife & 2 & 15,4 & 11 & 84,6 & 13 & 100,0 & \\
\hline Total & 19 & 12,1 & 138 & 87,9 & 157 & 100,0 & \\
\hline
\end{tabular}

após a exposição ao álcool, o nível de prolactina aumenta, enquanto a ocitocina diminui, tanto durante quanto depois da estimulação do seio da mãe. Porém, a ingestão de álcool é geralmente contraindicada no período de aleitamento, pois os supostos benefícios advindos dessa prática, como aumento da produção de leite e efeito tranquilizante nos bebês, não são efetivos $^{(24)}$. Pesquisa recente ${ }^{(25)}$ realizada em São Paulo encontrou, entre lactantes, relatos de que as bebidas alcoólicas são prejudiciais à lactação, não se constatando qualquer benefício galactogênico, ao contrário do mito popular.

O tabagismo, por sua vez, é um hábito socialmente aceito desde os tempos remotos, sendo prejudicial à criança que é amamentada, uma vez que esta entra em contato com as substâncias nocivas dos cigarros por meio do $\mathrm{LM}^{(4)}$. Nas lactantes estudadas, não se observou o uso de tabaco entre as mães que estavam amamentando e afirmaram consumir bebidas alcoólicas, diferentemente do que foi encontrado por Del Ciampo et $a l^{(4)}$, que relataram $26,8 \%$ de mães que consumiam álcool e também eram tabagistas. Rozov et al ${ }^{(26)}$, ao estudarem o hábito de fumar em 257 gestantes parturientes e seu entendimento acerca dos efeitos do fumo em fetos e lactentes, concluíram que $61 \%$ dos $\mathrm{RN}$ tinham risco de se tornarem fumantes passivos, caso as atitudes e os hábitos dos familiares não fossem modificados com a conscientização em pouco tempo.

Ao relacionar a frequência de aleitamento materno entre as mães consumidoras de álcool e as abstêmias, não se encontrou diferença estatística, como nos achados de Del Ciampo et al ${ }^{(4)}$, provavelmente devido ao efetivo trabalho realizado pelas equipes de saúde de Programas de Saúde da Família e da unidade participante deste estudo, estimulando e apoiando a prática do aleitamento materno. 
Ainda são escassos os dados da literatura que relacionam o consumo de alimentos durante a ingestão de bebidas alcoólicas no período da lactação. Nas lactantes estudadas, observou-se elevada frequência de consumo de petiscos (alimentos proteicos e sementes oleaginosas) durante a ingestão alcoólica. É importante destacar tal ocorrência, pois a ingestão de álcool associada a alimentos reduz a absorção alcoólica. O álcool é uma substância de rápida absorção e seu pico no sangue coincide com o no LM, em torno de 30 minutos a 1 hora $^{(19)}$. O consumo de álcool com alimentos pode retardar em até 90 minutos o pico do leite materno, com diferenças individuais consideráveis entre as mulheres ${ }^{(22)}$.

Uma possível limitação do presente estudo é o fato de ter sido usada uma amostra de conveniência, com seleção consecutiva, embora esta seja uma abordagem prática para grande parte dos projetos de pesquisa clínica. Dessa maneira, as conclusões do estudo podem não ser similares às que resultariam de uma amostra probabilística da população-alvo. Logo, atribuir baixa prevalência ao grupo de lactantes poderia ser uma limitação do modelo explicativo, com validade externa questionável. O delineamento transversal é adequado para fornecer informação sobre a proporção da população que consumia bebidas alcoólicas, com descrição das variáveis de estudo e seus padrões de distribuição. O número limitado

\section{Referências bibliográficas}

1. Santos-Torres MI, Vásquez-Garibay E. Food taboos among nursing mothers of Mexico. J Health Popul Nutr 2003;21:142-9.

2. Brasil - Ministério da Saúde. Pesquisa Nacional de Demografia e Saúde da Criança e da Mulher - PNDS 2006: dimensões do processo reprodutivo e da saúde da criança. Brasília: Ministério da Saúde, 2009.

3. Almeida CA, Del Ciampo LA, Ricco RG. Aleitamento materno: passagens e transferências mãe-filho. São Paulo: Atheneu; 2004.

4. Del Ciampo LA, Ricco RG, Ferraz IS, Daneluzzi JC, Martinelli Junior CE. Prevalence of smoking and alcohol consumption among mothers of infants under six months of age. Rev Paul Pediatr 2009;27:361-5.

5. Burgos MG, Medeiros MC, Bion FM, Pessoa DC. The effect of alcoholic beverages in nursing mothers and their impact on children. Rev Bras Saude Mater Infant 2002;2:129-35.

6. Tavares-do-Carmo MG, Nascimento-Curi CM. Effect of ethanol intake during lactation on the metabolism of dams and on pup development. Braz J Med Biol Res 1990;23:1161-3.

7. Carmo MG. Efeito da ingestão de álcool sobre o metabolismo lipídico em ratas lactantes [tese de doutorado]. São Paulo (SP): Escola Paulista de Medicina; 1993.

8. Giglia RC, Binns CW. Patterns of alcohol intake of pregnant and lactating women in Perth, Australia. Drug Alcohol Rev 2007;26:493-500.

9. Mennella JA, Gerrish CJ. Effects of exposure to alcohol in mother's milk on infant sleep. Pediatrics 1998;101:E2.

10. Tavares do Carmo MG, Oller do Nascimento CM, Martin A, Herrera E. Ethanol intake during lactation impairs milk production in rats and affects growth and metabolism of suckling pups. Alcohol 1999;18:71-6. de participantes diminui o poder estatístico da amostra e pode ter influenciado a falta de associação entre o consumo de álcool e algumas variáveis, como o aleitamento materno exclusivo e a escolaridade.

Em síntese, não se verificou consumo intenso de bebidas alcoólicas durante a lactação, e o álcool sempre esteve acompanhado da ingestão de alimentos (petiscos). Portanto, o consumo de álcool não representou um problema na amostra estudada. Entretanto, o uso de bebidas alcoólicas deve continuar sendo desencorajado durante a amamentação, e as mulheres com esse hábito precisam ser orientadas ao consumo esporádico, em pequena quantidade, combinado com a ingestão de alimentos. Os achados deste estudo representam uma contribuição original, alertando para os malefícios do álcool e sua possibilidade de veiculação através do LM. Desta maneira, preconizam-se mais investimentos públicos na prevenção dessa prática nos programas de pré-natal e puericultura.

\section{Agradecimentos}

À UFPE, pela oportunidade de conhecimento. À Prof $\mathrm{f}^{a}$ orientadora Goretti Burgos, pela dedicação e otimismo e por ter proporcionado muitos aprendizados. E aos pacientes, pela confiança.
11. Burgos MG, Bion FM, Campos F. Lactação e álcool: efeitos clínicos e nutricionais. ALAN 2004;54:25-35.

12. Pepino MY, Mennella JA. Advice given to women in Argentina about breastfeeding and the use of alcohol. Rev Panam Salud Publica 2004;16:408-14.

13. Giglia RC, Binns CW, Alfonso HS, Scott JA, Oddy WH. The effect of alcohol intake on breastfeeding duration in Australian women. Acta Paediatr 2008;97:624-9.

14. World Health Organization. Global status report on alcohol and health. Geneva: WHO; 2011

15. Saunders JB, Aasland OG, Babor TF, de la Fuente JR, Grant M. Development of the Alcohol Use Disorders Identification Test (AUDIT): WHO Collaborative Project on Early Detection of Persons with Harmful Alcohol Consumption-II. Addiction 1993;88:791-804.

16. Babor TF, Higgings-Biddle JC, Sauders JB, Monteiro MG. AUDIT: teste para identificação de problemas relacionados ao uso de álcool: roteiro para uso em atenção primária. Ribeirão Preto: Programa de Ações Integradas para Prevenção e Atenção ao Uso de Álcool e Drogas na Comunidade; 2003.

17. Laranjeira R, Pinsky I, Sanches M, Zaleski M, Caetano R. Alcohol use patterns among Brazilian adults. Rev Bras Psiquiatr 2010;32:231-41.

18. Maloney E, Hutchinson D, Burns L, Mattick RP, Black E. Prevalence and predictors of alcohol use in pregnancy and breastfeeding among Australian women. Birth 2011;38:3-9.

19. Brasil. Ministério da Saúde. Secretaria de Atenção à Saúde. Departamento de Ações Programáticas e Estratégicas [homepage on the Internet]. Amamentação e uso de medicamentos e outras substâncias [cited 2013 Mar 18]. Available from: http://bvsms.saude.gov.br/bvs/publicacoes/amamentacao_ uso_medicamentos_2ed.pdf 
20. Ichisato SM, Shimo AK. Breastfeeding and nutrition beliefs. Rev Lat Am Enfermagem 2001;9:70-6.

21. Parackal S, Ferguson E, Harraway J. Alcohol and tobacco consumption among 6-24-months post-partum New Zealand women. Matern Child Nutr 2007;3: 40-51.

22. Kachani AT, Okuda LS, Barbosa AL, Brasiliano S, Hochgraf PB. Breast-feeding: to which extent alcohol can affect the baby's health? Pediatria (São Paulo) 2008;30:249-56.

23. Vitolo MR, Patin RV, Bulow AC, Ganzerli M, Fisberg M. Conhecimentos e crendices populares de puérperas na prática da amamentação. Rev Nutr PUCCAMP 1994;7:132-47.
24. Mennella JA, Pepino MY, Teff KL. Acute alcohol consumption disrupts the hormonal milieu of lactating women. J Clin Endocrinol Metab 2005;90:1979-85.

25. Oliveira DR, Gomes PR, Bando AM, Gonçalves SR. Food beliefs in breastfeeding. A study among pregnant and lactating attended in a public maternity hospital in the city of São Paulo, Brazil. Arq Bras Cienc Saude 2011;36:67-71.

26. Rozov T, Fiss E, Catherino P, Perestelo MI, Nomura M. Hábito de fumar das gestantes e parturientes de um hospital universitário e seus conhecimentos sobre os efeitos do fumo em fetos e lactentes. Arq Med ABC 2004;29:28-36. 